\title{
Monitoring global traveling ionospheric disturbances using the worldwide GPS network during the October 2003 storms
}

\author{
Min Wang ${ }^{1,2,3}$, Feng Ding ${ }^{1}$, Weixing Wan ${ }^{1}$, Baiqi Ning ${ }^{1}$, and Biqiang Zhao ${ }^{1}$ \\ ${ }^{1}$ Division of Geomagnetism and Space Physics, Institute of Geology and Geophysics, Chinese Academy of Sciences, Beijing 100029, China \\ ${ }^{2}$ Wuhan Institute of Physics and Mathematics, Chinese Academy of Sciences, Wuhan 430072, China \\ ${ }^{3}$ Graduate University of Chinese Academy of Sciences, Beijing 100049, China
}

(Received February 21, 2006; Revised December 29, 2006; Accepted January 5, 2007; Online published June 8, 2007)

\begin{abstract}
The global traveling ionospheric disturbances (TIDs) during the drastic magnetic storms of October 29-31, 2003 were analyzed using the Global Position System (GPS) total electron content (TEC) data observed in the Asian-Australian, European and North American sectors. We collected the most comprehensive set of the TEC data from more than 900 GPS stations on the International GNSS Services (IGS) website and introduce here a strategy that combines polynomial fitting and multi-channel maximum entropy spectral analysis to obtain TID parameters. The results of our study are summarized as follows: (1) large-scale TIDs (LSTIDs) and mediumscale TIDs (MSTIDs) were detected in all three sectors after the sudden commencement (SC) of the magnetic storm, and their features showed longitudinal and latitudinal dependences. The duration of TIDs was longer at higher latitudes than at middle latitudes, with a maximum of about $16 \mathrm{~h}$. The TEC variation amplitude of LSTIDs was larger in the North American sector than in the two other sectors. At the lower latitudes, the ionospheric perturbations were more complicated, and their duration and amplitude were relatively longer and larger. (2) The periods and phase speeds of TIDs were different in these three sectors. In Europe, the TIDs propagated southward; in North America and Asia, the TIDs propagated southwestward; in the near-equator region, the disturbances propagated with the azimuth (the angle of the propagation direction of the LSTIDs measured clockwise from due north with $0^{\circ}$ ) of $210^{\circ}$ showing the influence of Coriolis force; in the Southern Hemisphere, the LSTIDs propagated conjugatedly northwestward. Both the southwestward and northeastward propagating LSTIDs are found in the equator region. These results mean that the Coriolis effect cannot be ignored for the wave propagation of LSTIDs and that the propagation direction is correlated with polar magnetic activity. Key words: Traveling ionospheric disturbances, Global Positioning System, gravity wave, total electron content.
\end{abstract}

\section{Introduction}

Most traveling ionospheric disturbances (TIDs) observed in the ionosphere are the response of the ionosphere to acoustic gravity waves (AGWs) (Hines, 1960). When the gravity waves reach ionospheric height, the neutral wind perturbations interact with the plasma via collisions. The motion of the charged particles, constrained to move along the magnetic field lines, carry them alternately to higher and lower altitudes. Transportation along the field lines results in electron density enhancements in some places along the wave fronts (where the electrons have moved in from above and below) and depletions in others. The regular enhancements and depletions of plasma density show traveling wave's properties, i.e. traveling ionospheric disturbances (Hooke and Schlegel, 1968; Tsugawa et al., 2003).

The observation and research of ionospheric perturbation and gravity waves have been an important subfield of ionospheric dynamics studies; they also constitute an important part of current research programs into space weather. In particular, the investigation of TIDs caused by storms provides data that furthers our understanding of the evolution

Copyright (c) The Society of Geomagnetism and Earth, Planetary and Space Sciences (SGEPSS); The Seismological Society of Japan; The Volcanological Society of Japan; The Geodetic Society of Japan; The Japanese Society for Planetary Sciences; TERRAPUB processes of energy transmission and conjugation from the high latitudes to the low latitudes. The techniques for observing ionosphere perturbations include incoherent scatter radar (Kirchengast et al., 1996), airglow imager (Ogawa et al., 2002; Shiokawa et al., 2002), ionosonde (Wan et al., 1995), HF Doppler (Wan et al., 1998), satellite beacon wave reception (Davies, 1980), and Global Positioning System (GPS), among others. Due to the high spatial and temporal resolution of GPS total electron content (TEC) data obtained from the International GNSS Service (IGS) network (Beutler et al., 1999), GPS TEC has, since the early 1990s, become the most widely used observation technique for investigating the properties of TIDs.

There are many studies that focus on using GPS TEC to study irregularities and perturbations in the ionosphere. Large-scale ionospheric structures and TIDs conjugate latitudinal enhancements were identified using GPS TEC difference maps during storm times (Ho, 1996). The Fregion Radio and Optical measurement of Nighttime TID (FRONT) campaign in Japan was conducted to clarify the physical mechanism of the TIDs in the F-region ionosphere. Based on these observations, several new features of the nighttime mid-latitude TIDs were determined, including the property of TIDs having a wide spectrum (from $3 \mathrm{~m}$ to 1000 $\mathrm{km}$ ) and the equatorward propagation of MSTIDs (Akinori, 


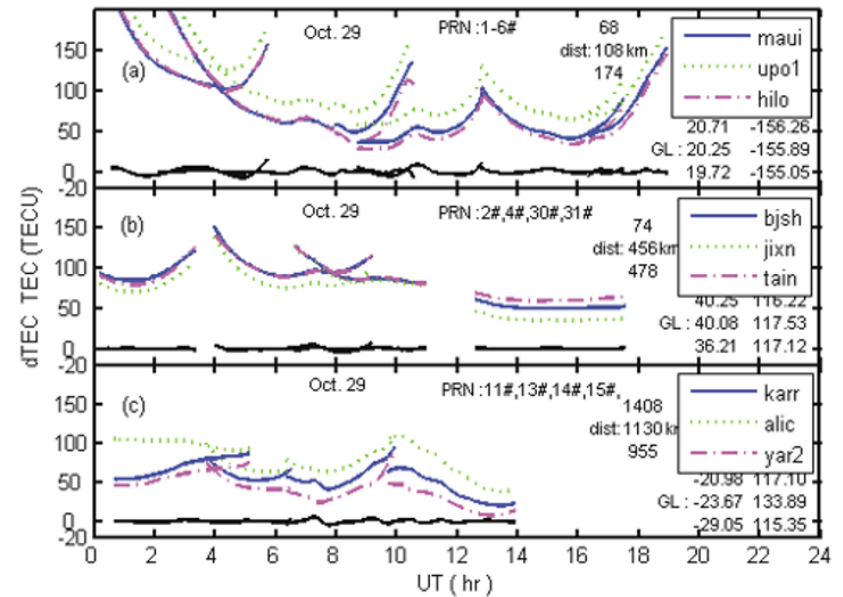

Fig. 1. In each figure, the three curves (upper) based on observed TEC data correspond to the three receivers of an array, which are indicated in the legend. The lines (lower) represent dTEC obtained from residues derived from multi-order polynomial fits TEC time series, and the magnitude stands for the amplitude of TID.

2002; Ogawa et al., 2002; Shiokawa et al., 2002). The FRONT-3 campaign observed MSTIDs in the airglow images with horizontal wavelengths of $100-400 \mathrm{~km}$ propagating southwestward in Japan and northwestward in Australia. A one-to-one correspondence of wave structures was found between the Northern and Southern Hemispheres, indicating strong electrodynamic coupling between the two hemispheres through the geomagnetic field line. In addition, LSTIDs propagating towards the equator with a spatial scale of approximately $1000 \mathrm{~km}$ were detected at these conjugate stations. The peak of LSTIDs in the Northern Hemisphere was 20 min earlier than that in the Southern Hemisphere, indicating that the observed LSTID was caused by a wave in the neutral atmosphere rather than by an electric field structure (Shiokawa et al., 2005). Tsugawa et al. (2003) and Shiokawa et al. (2005) used GPS TEC measured from more than 1000 GPS sites in the Japanese chain of islands to study the damping rate of LSTIDs during storm times and deduced that the damping was due to the ion-drag effect. Statistically, LSTID was also identified by a twodimensional GPS TEC map over Japan propagating southward with a $1000-\mathrm{km}$ scale, and some seasonal rules were proposed (Tsugawa and Saito, 2004; Shiokawa et al., 2002, 2005).

The majority of the investigations reported to date have emphasized the local characteristics of ionospheric perturbations. However, there is a subtle yet important trend in the research of the evolution processes of ionospheric distributions on the global scale. The purpose of this paper is to analyze the TIDs during the October 2003 magnetic storms in the Asia-Australia, European and North American sectors. By means of a multi-channel maximum entropy spectral analysis on the GPS TEC data from more than 900 GPS stations worldwide, we are able to provide a set of optimal TID parameters to describe the propagation features of TIDs during the superstorms.

This paper is organized as follows: Section 2 presents details on the method used here for processing GPS TEC

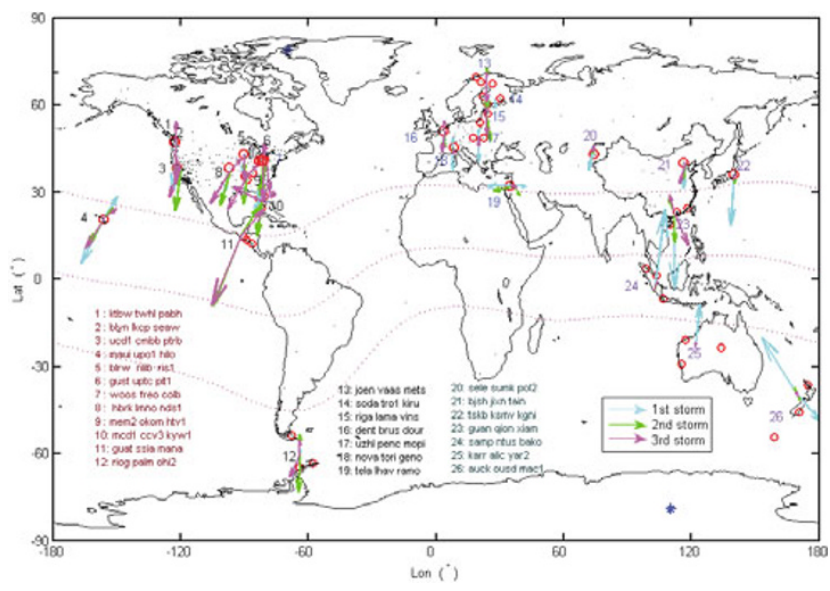

Fig. 2. The chosen locations of arrays, divided into three groups on the world map. The dots show the geographic distribution of more than 900 GPS receivers. One large circle represents one array and the small circles are stations of the other chosen arrays. The dashed lines denote dip latitudes $45^{\circ}, 0^{\circ}$ and $-45^{\circ}$. The “*” denotes the location of the magnetic pole. The vectors' diagram of TID propagation of each array during the three storms is also given. The amplitude of TID is represented by the length of arrow, and the propagation azimuth of TID is indicated by arrow's direction. The colors of the arrows, blue, green, and purple, are associated to the first storm, second storm, and third storm, respectively. For each array, the long arrow of the same color is the dominant propagation azimuth, while the short arrow is the minor azimuth.

data; Section 3 introduces the statistical results from different global sectors together with discussions; Section 3 presents our conclusions.

\section{GPS TEC Data Processing \\ 2.1 Selection of GPS stations}

The GPS TEC data used in this study were obtained from the data center of the IGS network. We selected 26 sets of arrays with three GPS stations for each array; in this way we can acquire the data using correlation analysis method to pick up the ionospheric disturbances in different areas. In order to avoid the problem of phase integer cycle ambiguity in the time series of GPS TEC data, we adopted the constraint that the distance between the different stations in the same array should be less than the half of the horizontal wavelength of the TIDs. Based on Hunsucker (1982), Hocke and Schlegel (1996) distinguished between LSTIDs with a period greater than $30 \mathrm{~min}$ and moving faster than $300 \mathrm{~m} / \mathrm{s}$ and MSTIDs with shorter periods (from 10 to 50 $\mathrm{min}$ ) and moving slower (50-300 m/s); accordingly, the distances between selected stations should be as small as possible and set at no more than $1500 \mathrm{~km}$ in order to accurately estimate the parameters of the disturbance propagation. Since the stations are dense at mid-latitudes, the distances among most selected stations are less than $200 \mathrm{~km}$. However, due to the sparseness of the observational network in the Southern Hemisphere, the constrained distances among stations there are usually more than $1000 \mathrm{~km}$ so that we can only focus on LSTIDs with a horizontal wavelength of about $2000 \mathrm{~km}$.

Figure 1 represents the waveform of TEC disturbances observed from three different arrays on October 29, which is the first day of the storm. As can be seen from each panel, 


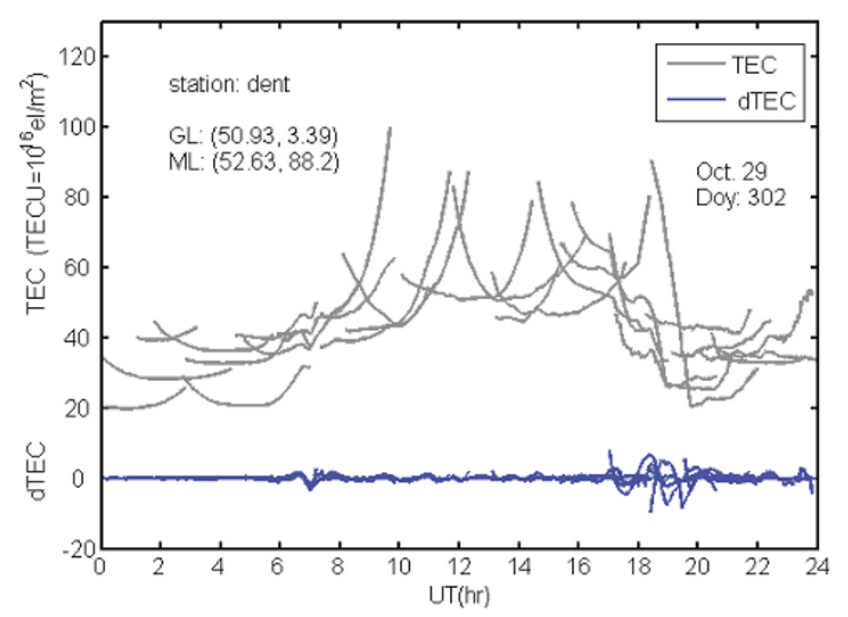

Fig. 3. Background trends were removed using residues obtained from polynomial fits. Observed slant TEC data from 31 GPS satellites are indicated by the gray solid curves (upper). The solid lines (lower) represent dTEC obtained from residues derived from multi-order polynomial fits TEC time series.

the same satellite at the same time interval has a very similar waveform. Figure 1(a)-(c) also shows the satellite number $(\mathrm{PRN})$, geographic latitude and geographic longitude (GL) and their stations' distances (dist) in each array. It is apparent that although the distances among these arrays are different, the curves of the observed TEC time series are very similar. As Fig. 1(c) illustrates, although the distances among the stations are as great as $900-1400 \mathrm{~km}$, the waveform of the observed TEC time series still maintains a good correlation. Through extensive experimentation on the time series from each array, we were able to determine that if the distance among the chosen stations is less than $200 \mathrm{~km}$, we can obtain a high coherency of wave parameters where the correlation coefficient is larger than 0.98; if the distance is between $200 \mathrm{~km}$ and $600 \mathrm{~km}$, the correlation coefficient is about 0.95 . Based on these rules of selection, we analyzed the time series observed by each array that we selected on the global scale and obtained accurate wave parameters using three-channel maximum entropy spectral analysis after filtering.

Figure 2 shows the locations of the 26 chosen sets of GPS arrays, which are numbered from Array 1 to 26 , respectively, and divided into three groups on the world map. As shown in this figure, there are more GPS stations in the North American, European and Asian sectors than over the sea and Southern Hemisphere.

\subsection{Method of calculating propagation parameters of TIDs from GPS TEC}

The four steps of our primary three-phase data processing are as follows:

(1) We converted GPS observation files in Renix format into slant TEC time series in ASCII format and removed the outliers and wild points.

(2) Background trends were filtered out from the time series of TEC. We applied a sliding window with a duration of $2-3 \mathrm{~h}$ to the original time series of TEC data. Background trends of the time series were removed from these sliding windows using residues obtained from 2- to 3-order polynomial fits (Ding et al., 2004) and a band-pass filter with the passband $(60 /(2 \times 3600), 60 /(10 \times 60))$. Note that there are many issues involved in the background trends of slant TEC, such as TEC diurnal variation, TEC variations relative to the elevation angle of the line of sight, TEC variation with latitudes and longitudes, and Doppler Effect due to satellite motion of GPS, among others. Figure 3 shows the original TEC time series (upper) observed at station dent $\left(50.93^{\circ} \mathrm{N}, 3.39^{\circ} \mathrm{E}\right)$ on October 29,2003 and the time series of TEC fluctuation (dTEC) after filtering that is used to describe ionosphere disturbances obtained from residues derived from multi-order polynomial fits of the original TEC time series in sliding windows. This figure illustrates that our method is very effective in showing that the intense period of disturbance variation of TEC appears between 1800 UT-2000 UT (1800-2000 LT) instead of in the afternoon (about 1400 LT) when the strongest range of TEC diurnal variation occurs.

(3) By adopting the three-channel maximum entropy method (Strand, 1977), we derived the main frequency and phase differences between dTEC fluctuation time series.

$$
\left(f, \phi_{21}, \phi_{31}\right)=\operatorname{MEM}\left(I_{1}, I_{2}, I_{3}\right)
$$

where $I_{1}, I_{2}$, and $I_{3}$ are the dTEC fluctuation time series observed at the same time at three stations, MEM is the maximum entropy method, $f$ is wave frequency, and $\phi_{21}$, $\phi_{31}$ are phase differences between the time series observed at the three stations. Galileo transformation was applied to eliminate the error caused by the Doppler shift in the dTEC time series due to the movements of GPS satellites (Wan et al., 1997).

$$
\omega_{\mathrm{wave}}=\omega_{\mathrm{observe}}+\vec{k}_{\mathrm{wave}} \cdot \vec{u}_{\mathrm{ipp}}
$$

where $\vec{u}_{\text {ipp }}$ represents the horizontal speed of the movement of the ionospheric pierce point.

(4) Finally, we identified TID events and evaluated the propagation parameters of TIDs. Assuming that the surface of the Earth around the observation points is a plane; a rectangular plane coordinate system is set with the origin at the observation station, in a way that the wave parameters satisfies:

$$
\left\{\begin{array}{l}
k_{x} x_{21}+k_{y} y_{21}=\phi_{21} \\
k_{x} x_{31}+k_{y} y_{31}=\phi_{31}
\end{array}\right.
$$

where $k_{x}$ and $k_{y}$ are unknown horizontal wave numbers. Terms $\left(x_{21}, y_{21}\right)$ and $\left(x_{31}, y_{31}\right)$ are the relative positions of the observation points. Equation (3) yields the solution of $k_{x}$ and $k_{y}$ :

$$
k_{x}=\frac{y_{21} \phi_{31}-y_{31} \phi_{21}}{x_{31} y_{21}-x_{21} y_{31}} k_{y}=\frac{x_{21} \phi_{31}-x_{31} \phi_{21}}{x_{21} y_{31}-x_{31} y_{21}}
$$

Consequently, we obtain the horizontal phase speed $v_{p h}$ and wave propagation azimuth $\sigma$ :

$$
\begin{gathered}
v_{p h}=2 \pi f / \sqrt{k_{x}^{2}+k_{y}^{2}} \\
\sigma=\arctan \left(k_{x} / k_{y}\right) \\
T=1 / f
\end{gathered}
$$


Table 1. The parameters for TIDs propagation from 26 chosen arrays in the 3 storms.

\begin{tabular}{|c|c|c|c|c|c|c|c|c|c|c|c|c|c|c|c|c|}
\hline \multirow[t]{2}{*}{ Region } & \multirow[t]{2}{*}{ No } & \multirow[t]{2}{*}{ GPS array } & \multirow{2}{*}{$\begin{array}{c}\text { Glat, Glon } \\
\left({ }^{\circ}\right)\end{array}$} & \multirow{2}{*}{$\begin{array}{c}\text { Mlat, Mlon } \\
\left({ }^{\circ}\right)\end{array}$} & \multicolumn{3}{|c|}{ Amplitude (TECU) } & \multicolumn{3}{|c|}{ Period $\pm 15(\mathrm{~min})$} & \multicolumn{3}{|c|}{$\mathrm{Vph}\left(\mathrm{m} \cdot \mathrm{s}^{-1}\right)$} & \multicolumn{3}{|c|}{ Azimuth $\left(^{\circ}\right)$} \\
\hline & & & & & 1 st & 2nd & $3 \mathrm{rd}$ & 1 & 2 & 3 & $1 \mathrm{st}$ & 2nd & $3 \mathrm{rd}$ & 1 st & 2nd & $3 \mathrm{rd}$ \\
\hline \multirow{3}{*}{$\begin{array}{l}\text { High latitude } \\
\text { (Europe) }\end{array}$} & 13 & joen vaas mets & 62,28 & 58,119 & \pm 12 & \pm 12 & & 60 & & $100-500$ & & & 180 & & & \\
\hline & 14 & soda tro1 kiru & 68,24 & 63,120 & \pm 15 & \pm 12 & \pm 12 & 50 & 60 & 45 & $200 \pm 200$ & $<200$ & $<200$ & 180 & $180(360)$ & $180(360)$ \\
\hline & 15 & riga lama vlns & 56,24 & 54,110 & \pm 3.5 & \pm 12 & \pm 10 & 60 & 60 & 60 & $200-400$ & $100-300$ & $100-300$ & 175 & 170 & $180(360)$ \\
\hline \multirow{9}{*}{$\begin{array}{c}\text { Mid-Lat } \\
\text { (North Am) }\end{array}$} & 1 & ktbw twhl pabh & $47,-122$ & $53,-63$ & \pm 10 & \pm 22 & \pm 23 & 42 & 42 & 45 & $200-400$ & $<250$ & $200-400$ & 180 & 180 & $180(360)$ \\
\hline & 2 & blyn lkcp seaw & $48,-122$ & $54,-63$ & \pm 12 & \pm 22 & \pm 21 & 38 & 33 & 38 & $150(450)$ & $400 \pm 200$ & 150 & 185 & 180 & $180(360)$ \\
\hline & 3 & ucd $1 \mathrm{cmbb}$ ptrb & $38,-120$ & $45,-59$ & \pm 1 & \pm 24 & \pm 18 & 60 & 48 & 48 & $200-400$ & $200-400$ & $<200$ & 185 & 190 & $210(330)$ \\
\hline & 5 & blrw nlib ris1 & $43,-90$ & $53,-24$ & \pm 4 & \pm 9 & \pm 14 & 42 & 45 & 42 & $250(600)$ & $200 \pm 100$ & $200 \pm 100$ & 190 & 180 & 180 \\
\hline & 6 & gust uptc pit1 & $41,-80$ & $52,-12$ & \pm 10 & \pm 20 & \pm 19 & 45 & 45 & 70 & $200 \pm 100$ & $150 \pm 100$ & $250 \pm 100$ & 190 & 190 & $170(360)$ \\
\hline & 7 & woos freo colb & $40,-81$ & $51,-13$ & \pm 10 & \pm 18 & \pm 22 & 42 & 45 & 45 & $350 \pm 100$ & $<200$ & $200-800$ & 190 & 190 & $180(360)$ \\
\hline & 8 & hbrk lmno nds1 & $37,-97$ & $47,-30$ & \pm 5 & \pm 20 & \pm 23 & 50 & 70 & 48 & $400 \pm 200$ & $200-600$ & $150 \pm 100$ & 200 & 200 & $220(50)$ \\
\hline & 9 & mem 2 okom htv 1 & $35,-89$ & $45,-22$ & \pm 3 & \pm 15 & \pm 23 & 48 & 45 & 45 & $400 \pm 200$ & $200 \pm 100$ & $200 \pm 100$ & 195 & 190 & $180(340)$ \\
\hline & 10 & $\operatorname{mcd} 1 \operatorname{ccv} 3$ kyw 1 & $27,-82$ & $38,-13$ & \pm 2 & \pm 20 & \pm 23 & 50 & 45 & 45 & $550 \pm 200$ & $200-800$ & $200-400$ & 240 & 190 & $270(60)$ \\
\hline \multirow{3}{*}{$\begin{array}{l}\text { Mid-Lat } \\
\text { (Europe) }\end{array}$} & 16 & dent brus dour & 51,4 & 52,88 & \pm 2 & \pm 10 & \pm 12 & 48 & 48 & 48 & $300 \pm 100$ & $200 \pm 100$ & $200 \pm 100$ & 185 & 185 & $190(360)$ \\
\hline & 17 & uzhl penc mopi & 48,20 & 47,102 & \pm 4 & \pm 2 & \pm 2 & 50 & 50 & 50 & $200-800$ & $<400$ & $<100$ & $180(360)$ & 180 & $180(360)$ \\
\hline & 18 & nova tori geno & 45,8 & 46,90 & \pm 5 & \pm 1 & \pm 1 & 48 & 48 & 48 & $200 \pm 100$ & $400-800$ & $200 \pm 100$ & $180(360)$ & 180 & 185 \\
\hline \multirow{3}{*}{$\begin{array}{c}\text { Mid-Lat } \\
\text { (Asia) }\end{array}$} & 20 & sele sumk pol2 & 46,74 & 33,152 & \pm 6 & \pm 3 & \pm 2 & 48 & 48 & & $600 \pm 200$ & $200 \pm 200$ & & 195 & 185 & \\
\hline & 21 & bjsh jixn tain & 40,117 & $29,-173$ & \pm 6 & \pm 10 & \pm 12 & 50 & 50 & 50 & $500-800$ & $200-600$ & $300-600$ & 185 & 190 & 200 \\
\hline & 22 & tskb ksmv kgni & 36,140 & $26,-152$ & \pm 12 & \pm 5 & \pm 3 & 55 & 45 & 45 & $400-600$ & $200-400$ & $200-400$ & 185 & 170 & 185 \\
\hline \multirow{5}{*}{$\begin{array}{l}\text { Low latitude } \\
\text { and equator } \\
\text { regions }\end{array}$} & 4 & maui upo1 hilo & $21,-156$ & $21,-89$ & \pm 13 & \pm 18 & \pm 22 & 45 & 45 & 45 & $200-400$ & $0-300$ & $200 \pm 100$ & $215(40)$ & $220(50)$ & $220(60)$ \\
\hline & 11 & guat ssia mana & $15,-91.5$ & $24,-21$ & & \pm 45 & \pm 45 & & 45 & 40 & & 200 & $100 \pm 100$ & & $210(40)$ & $210(50)$ \\
\hline & 19 & tela lhav ramo & 32,36 & 28,111 & \pm 8 & \pm 15 & \pm 2 & 45 & 45 & 45 & $200 \pm 200$ & $400-800$ & $100 \pm 100$ & $270(90)$ & $260(130)$ & $100(270)$ \\
\hline & 23 & guan qion xiam & 23,112 & $12,-176$ & \pm 18 & \pm 17 & \pm 21 & 48 & 48 & 45 & $350 \pm 200$ & $400 \pm 200$ & $400 \pm 200$ & 180 & $180(330)$ & $150(340)$ \\
\hline & 24 & samp ntus bako & $-3,103$ & $-8,170$ & \pm 19 & \pm 2 & \pm 4 & 48 & 48 & 48 & $700-900$ & $250-600$ & $400-800$ & 10 & $220(20)$ & $20(230)$ \\
\hline \multirow{3}{*}{$\begin{array}{c}\text { Southern } \\
\text { Hemisphere }\end{array}$} & 12 & riog palm ohi2 & $-60,-64$ & $-43,3$ & \pm 2 & \pm 22 & & 45 & 45 & 45 & $200-900$ & $200-900$ & & $185(20)$ & $182(360)$ & \\
\hline & 25 & karr alic yar2 & $-23,122$ & $-32,-171$ & \pm 10 & \pm 1 & \pm 1 & 48 & 50 & 62 & $800 \pm 200$ & $600-800$ & $600 \pm 200$ & 8 & $360(210)$ & $360(210)$ \\
\hline & 26 & auck ousd mac1 & $-40,170$ & $-50,-107$ & \pm 18 & \pm 5 & \pm 1 & 65 & 48 & 62 & $400-600$ & $400 \pm 200$ & $400 \pm 200$ & $320(130)$ & $330(150)$ & $330(150)$ \\
\hline
\end{tabular}

The experimental results showed that the suggested method of residues obtained from the multi-order polynomial fits gives a good result in terms of acquiring the variation of perturbance within a short time series by removing the long-term trend. The MEM spectral analysis also assures the acquisition of a high-resolution frequency domain and facilitates our analysis of the qualities of wave propagation parameters in the time and frequency domains. This in turn provides the accurate value of the propagation parameters of TIDs.

\subsection{Identification of TID events}

We applied a sliding window with a duration of $2-3 \mathrm{~h}$ to the original dTEC time series. This generates a sequence of a shorter time series within the 2 - to $3-\mathrm{h}$ period. The length of the chosen sliding window was set to be longer than the estimated period of wave disturbance so that the time series of $2-3 \mathrm{~h}$ is long enough to contain at least one cycle of TID. The three-channel MEM was then applied to the generated shorter time series. This yielded a sequence of TIDs propagation parameters which vary with time.

The main reason for determining these parameters is as follows. It is necessary to determine whether there is a most frequent and dominant value in the distribution function of the period, in horizontal phase velocity, and in the azimuth of the ionospheric disturbances during the observation intervals. If such a dominant value exists, the ionospheric disturbances with the similar waveform among the time series, which are detected by the three GPS stations of one array, can be regarded as one TID, and the distances between the chosen stations are reasonable. As a result, the mean value and standard deviation of the computed peak values of period, horizontal phase velocity, and azimuth could be used to express their true values and the dominant range of variation at the array area, respectively.

Using the method described above, we can auto-identify the duration of the disturbances and merely calculate the part of the existing disturbances. We divided the perturbation of 3 days into three intervals. The first duration was 0400-1400 UT October 29; the second duration was from 1200 UT October 29 to 0800 UT October 30; the third duration was from 0600 UT October 30 to 1300 UT October 31. According to the geomagnetic indices Dst and AEt, there were three major groups of storms in existence. The propagation parameters of the time series in terms of the disturbances in the three intervals are statistically illustrated in Table 1 and Fig. 4. The examples, which consist of distribution plots and a histogram of the TIDs parameters (period, the horizontal velocity, and the azimuth) of about nine arrays ((a)-(c) are from North America, Europe and AsiaAustralia, respectively) calculated by MEM, are exhibited in Fig. 4.

In general, if there are wave-like structures in the time series, this analysis reveals the observed period, horizontal phase speed, and propagation direction of the TIDs. If a steady wave field is passing, the wave parameters for the components of the field should remain steady for the duration of that field (Ding et al., 2004). Because of the continuous observation of GPS TEC data, we can obtain mean wave parameters on each day during the storm time using Eqs. (4) -(7).

\section{Statistical Results and Discussion}

A drastic burst of solar activity - the largest since 1990occurred during the period October 29 to November 01, 2003. This triggered three sets of storms, which were accompanied by a series of ionospheric storms. A threefold 

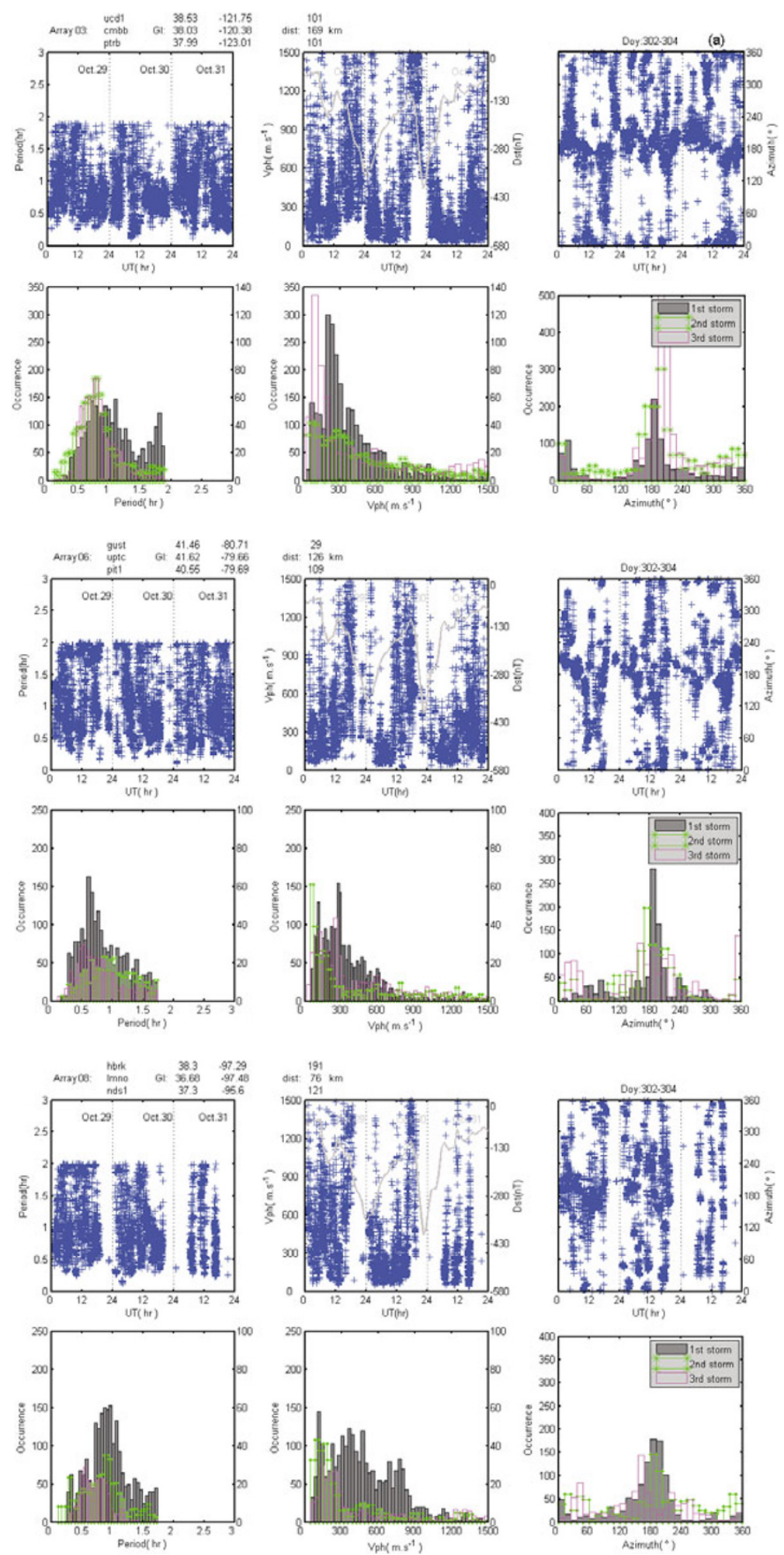

Fig. 4(a). The distribution plots and histogram of wave parameters derived from three-channel maximum entropy spectral analysis. The detected TID periods (hours, first row), velocities (m/s, second row), and azimuth (degree, third row) for North America (a), Europe (b), and Asia (c), respectively. The first storm was at 0400-1400 UT October 29; the second storm, 1600 UT October 29 to 1000 UT October 30; the third storm, 1400 UT October 30 to 1000 UT October 31.

decrease in the Dst time series corresponds to the three major groups of storms. During these three superstorms, the maximum Kp reached 9, the Dst index declined to a minimum of $-400 \mathrm{nT}$, and the AE index reached a maximum of $5000 \mathrm{nT}$.

Figure 5 presents the time series of ionospheric distur- bances acquired from GPS array 7 (woos freo colb) $\left(40^{\circ} \mathrm{N}\right.$, $81^{\circ} \mathrm{W}$ ) during the period October 29 to November 1, 2003. This figure also illustrates that the amplitude of ionospheric disturbances during the main phase of the storms is more obvious than those at other times. It also shows that there is a high correlation between the time series of ionospheric 

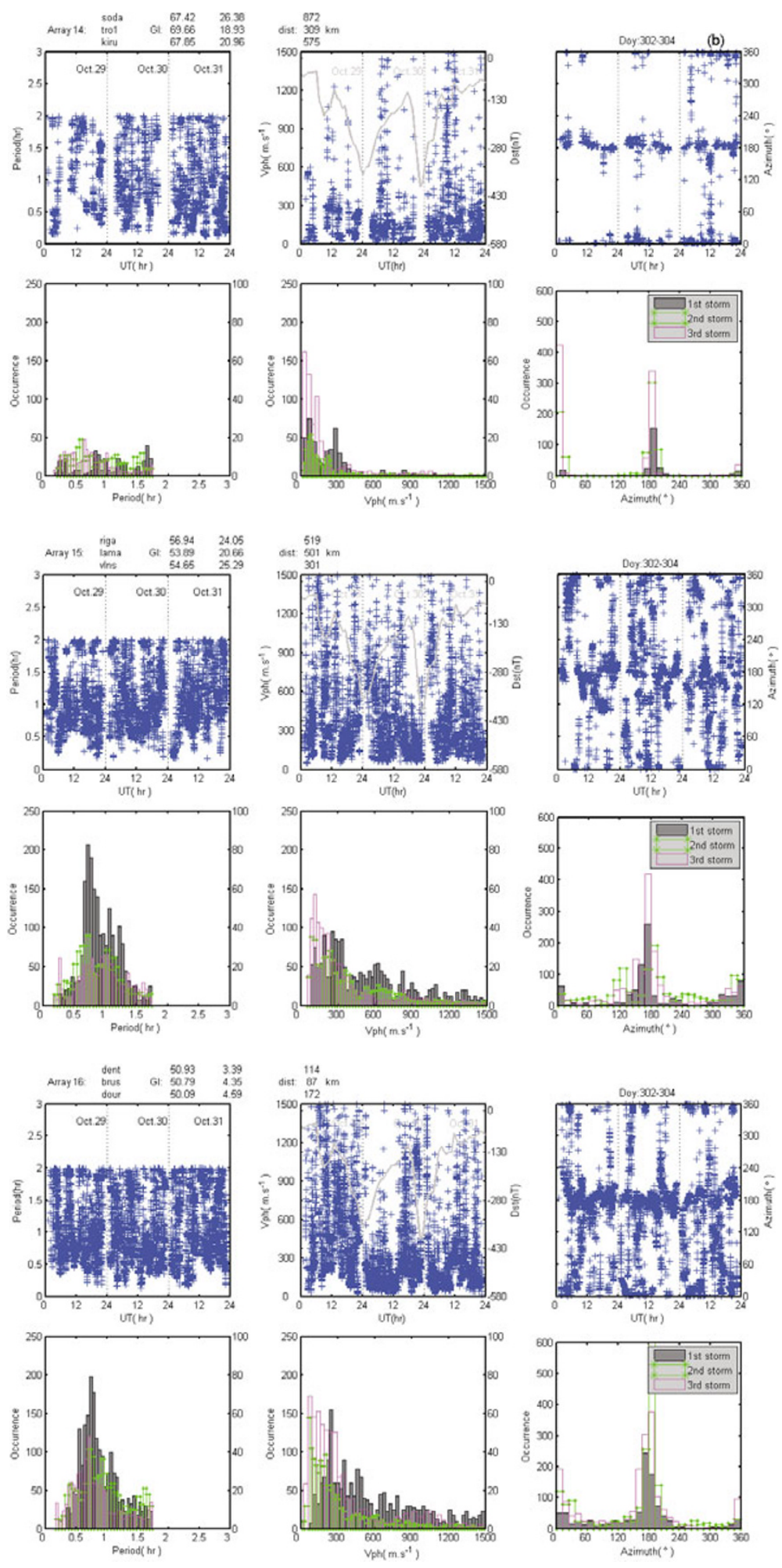

Fig. 4(b). (continued).

disturbances and the variations in the Dst and AE index and that there is a slight lag or advance corresponding to the latitudes of different regions. Although this agrees with the concept of the geomagnetic control of the TID amplitude, it does not correlate with the absolute level of Dst, but with the value of the time derivative of Dst. The phenomenon is also illustrated by Afraimovich et al. (2001).

We applied the methods described in Section 2 to an anal- ysis of the TEC data from 26 sets of GPS receiver arrays located in the American, European, Asian and Australian sectors and calculated the propagation parameters, including amplitude, period, horizontal phase velocity, and azimuth, of the ionosphere disturbance. The parameters for TIDs propagation derived from 26 chosen arrays data are presented in Table 1. 

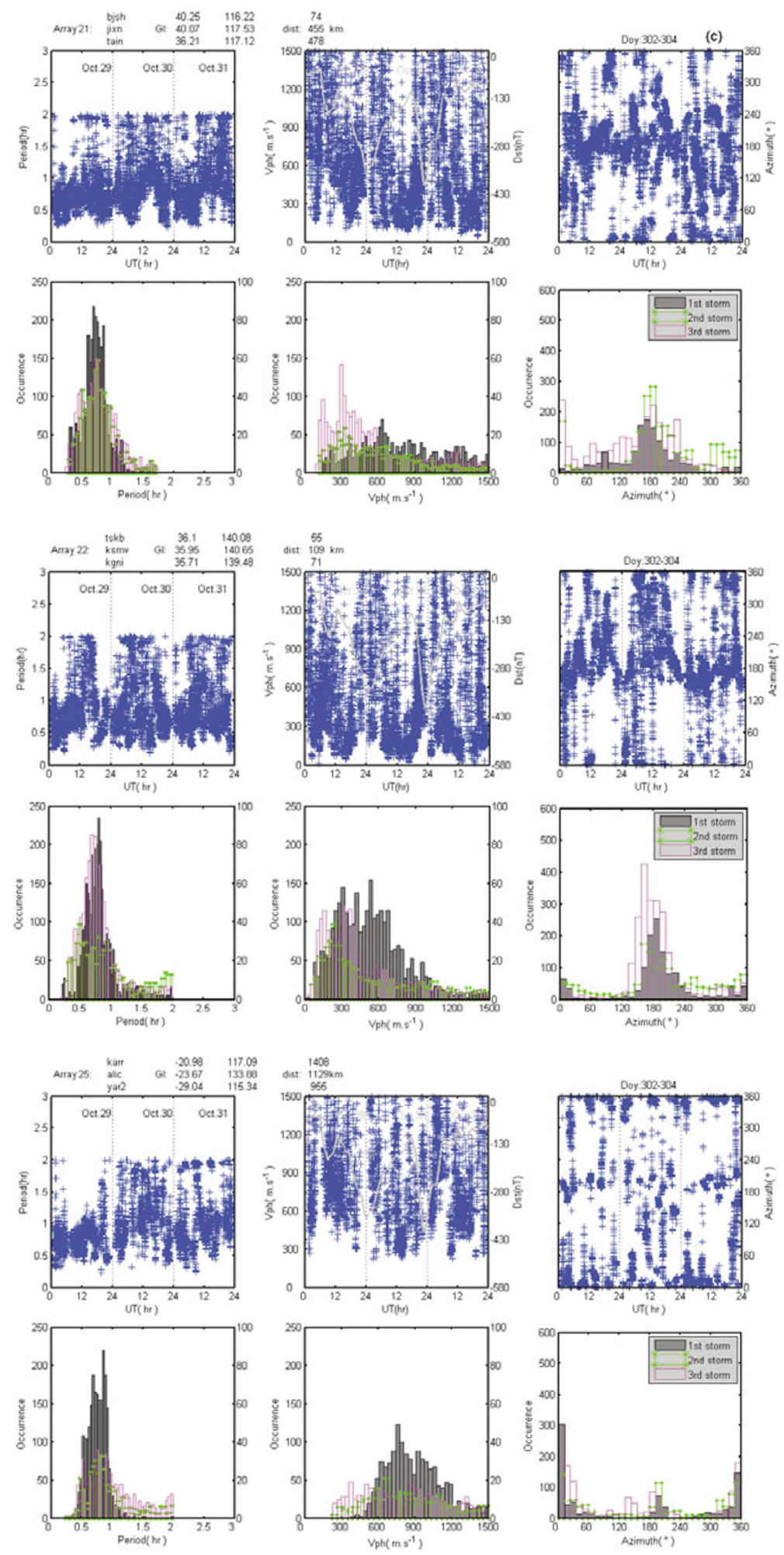

Fig. 4(c). (continued).

\subsection{TIDs observed in high latitude}

We selected three sets of arrays-13, 14, and 15-in the European sector at the Northern Hemisphere high latitudes to calculate the values of the MSTID and LSTID propagation parameters during the three major storms in October 29 to October 31. Figure 2 shows the locations of these arrays. Following the sudden commencement (SC) of the magnetic storm at 0612 UT on October 29, the TIDs at the geographical latitudes $60-75^{\circ} \mathrm{N}$ were detected with maximum amplitudes of $17 \mathrm{TECU}$, which were larger than those at middle latitudes $50-60^{\circ} \mathrm{N}$. The LSTIDs with a mean period of $55 \mathrm{~min}$ were detected as wave-like structures, propagated in the equatorward direction with a horizontal velocity of about $400-600 \mathrm{~m} / \mathrm{s}$ and southward phase progression as the main azimuth of $180^{\circ}$. In addition, the concomitant MSTIDs with a period of $45 \mathrm{~min}$ were also detected with 
Table 2. Average evaluations of wave parameters at mid-latitudes.

\begin{tabular}{ccccccc}
\hline & $\begin{array}{c}\text { Amplitude } \\
(\text { TECU })\end{array}$ & $\begin{array}{c}\text { Period } \\
(\mathrm{min})\end{array}$ & & $\begin{array}{c}\text { Vph } \\
\left(\mathrm{ms}^{-1}\right)\end{array}$ & $\begin{array}{c}\text { Azimuth } \\
\left({ }^{\circ}\right)\end{array}$ \\
\hline North-Am & \pm 20 & $\sim 46$ & $200-600$ & $200 \pm 100$ & $200 \pm 100$ & 187.6 \\
Europe & $\pm 10-15$ & $\sim 49$ & $250 \pm 100$ & $300-400$ & $200 \pm 100$ & 184.0 \\
Asia & \pm 10 & $\sim 49$ & $400-700$ & $200-500$ & $200-500$ & 187.5 \\
\hline
\end{tabular}

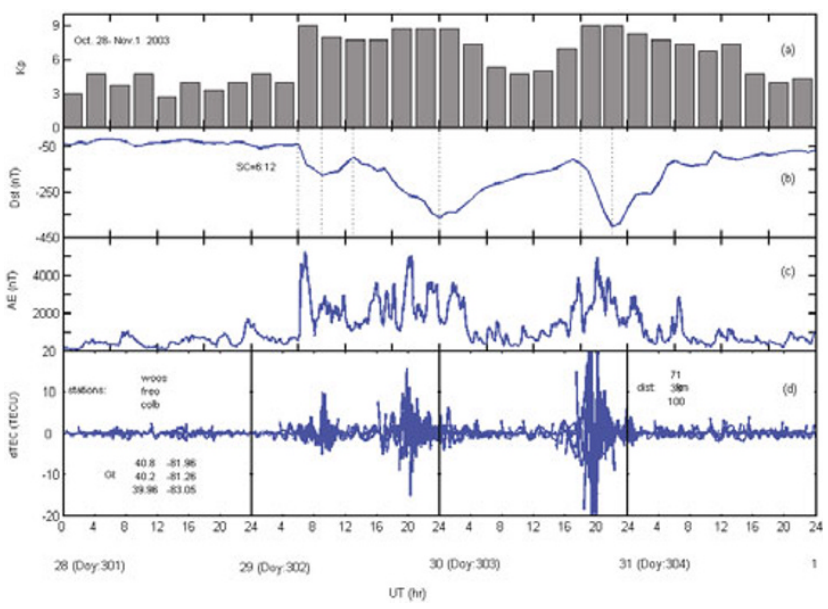

Fig. 5. Variations of the geomagnetic indices Kp (a), Dst (b), and AE (c) on October 28 to November 1, 2003. (d) shows the variation of TEC during the geomagnetic storms observed at mid-latitudes (GPS array no.7). Dashed lines in the second panel indicate times that Dst values started to decrease to their minimums.

a horizontal velocity of less than $300 \mathrm{~m} / \mathrm{s}$ to propagate due southward. The maximum traveling time of TIDs is around $12-16 \mathrm{~h}$ in this area.

\subsection{LSTIDs observed in middle latitude}

As described by Lin et al., equatorial ionization anomaly (EIA) expanded to very high latitudes during the entire storm period, with a large increase in TEC immediately after the storm started that is detected in Northern America. This illustrated the occurrence of a strong plasma fountain effect, perhaps caused by a penetration electric field from the high to low latitude, and a strong upward $\mathrm{E} \times \mathrm{B}$ drift in the ionosphere lifted the ionosphere layers (Foster and Rideout, 2005; Lin et al., 2005; Zhao et al., 2005). All of these led to larger amplitudes in the ionosphere disturbances in the American sector compared with those in the European and Asian sectors.

From the results of spectral analysis (Fig. 4) derived from 14 GPS arrays (1-3, 5-8, 16-22) at the mid-latitudes, we obtained the parameters of ionosphere disturbances in North America, Europe, and Asia, shown in Table 1. It can be seen that a small quantity of MSTIDs with scales of 20-40 min correspond to the enhancement of LSTIDs with scales from $40 \mathrm{~min}$ to $2 \mathrm{~h}$, with the obvious dominating MSTID due southward or southwestward direction independent of the scales. Moreover, in this case, an increase in the intensity of medium-scale and small-scale irregularities is caused by the dissipation process of intense LSTIDs of auroral origin, accompanied by the appearance of ionospheric irregularities in a wide range of scales, including MSTIDs and small-scale ones. These phenomena were also observed by Afraimovich et al. (2006). The propagation directions of those ionospheric disturbances had shifted somewhat clockwise from the south, resulting in a NW-SE phase surface and a southwestward movement in the Northern Hemisphere. Shiokawa et al. (2003) suggested that the electrodynamic instability in the ionosphere could cause southwestward MSTIDs that are generated by an oscillating electric field in the ionosphere (Fukao et al., 1991; Garcia et al., 2000). However, there is still no evidence to show that the electrodynamic instability caused these large-amplitude LSTIDs. Thus, we believe that the westward deflection of those disturbances, including LSTIDs and medium-scale and small-scale irregularities caused by the process of dissipation of intense LSTIDs during the three storms, is the result of both the westward-blowing thermospheric winds and the Coriolis force effect attributed to the Earth's rotation. This means that the Coriolis effect cannot be ignored for the wave propagation of LSTIDs (Sawako et al., 1980). This will be discussed further in Section 3.3.

The results of the spectral analysis reinforced the results indicating an increase in intense LSTIDs during the propagation. Figure 6 illustrates the waveform of the disturbance time series based on data from the ten arrays at the middle latitudes. This waveform reveals that in the North American sector, both the value of TEC and the amplitudes of disturbance are largerr than those in Europe and Asia after the storm SC.

From the average parameters of ionosphere disturbances in North America, Europe, and Asia presented in Table 2, the average period of disturbances is approximately $46 \mathrm{~min}$. There is an obvious dominating southwestward direction for ionospheric disturbances, with an average azimuth of $187.6^{\circ}$, and the southward propagation direction deviated about $7^{\circ}$ to the west.

Meanwhile, the propagation parameters in the European and Asian sectors differ considerably from those in North America. The amplitudes of the disturbances in Europe are larger than those in Asia. The ionospheric disturbances observed in Europe were characterized by an average period of $49 \mathrm{~min}$ and a propagation azimuth of $184^{\circ}$ during the three storms. While in Asia, the scale keeps on $49 \mathrm{~min}$ for period and $187.5^{\circ}$ for mean azimuth of those ionospheric disturbances during the three storms.

\subsection{LSTIDs observed in at low latitudes and the equa- tor regions}

The data from GPS arrays 4, 11, 19, 23, and 24 were selected to display the propagation of LSTIDs at the low latitudes and equator regions. There are abundant ionospheric disturbances in these regions. The phenomena of disturbances are complex: the amplitudes peak from 13 to 45 TECU on October 29, and the duration is longer than that at the mid-latitudes and high latitudes. Figure 7 illustrates the 

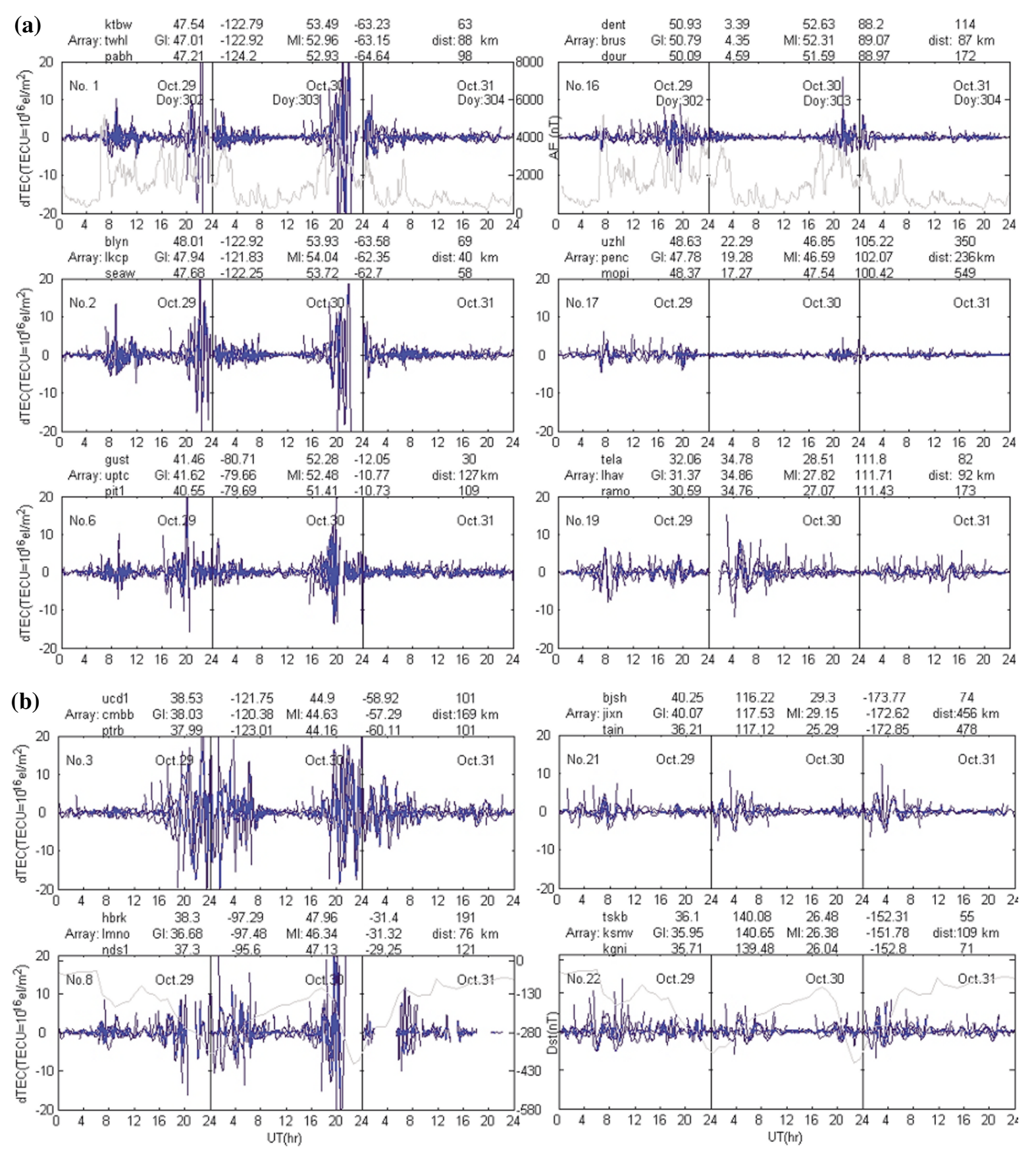

Fig. 6. Variations in dTEC time series at mid-latitudes (the range of geographic latitudes from $30^{\circ}$ to $60^{\circ}$ ) during the October $29-31$ magnetic storms. The locations of these arrays are given above each panel. GL: geographic latitude and geographic longitude, ML: geomagnetic latitude and geomagnetic longitude, dist: the distances between two stations. The geomagnetic indices AE and Dst are also displayed by gray lines.

waveform of disturbances according to five arrays at low latitudes. The ionospheric disturbances of the middle and low latitudes in the Eastern Hemisphere occurred on October 28 (301st day) before the SC of the storm, but they were not detected in North America due to high geomagnetic latitudes. The big fountain effect increased the TEC in both hemispheres before the SC. Nevertheless, compared with October 29, the TEC had extended to slightly higher latitudes but had not spread to North America, and the westward propagation was not as significant on October 28. The values of the horizontal phase velocity of the ionospheric disturbances are diverse in these respective regions. The two dominant directions of propagation can be observed at the low latitudes and equatorial region. The southward propagation direction is deflected by $35^{\circ}$ to the west, the while propagation direction of the northward disturbances are deflected by $35 \pm 20^{\circ}$ to the east. We believe that the deflection to west (Saito et al., 1998; Ogawa et al., 2002; Shiokawa et al., 2003, 2005; Tsugawa et al., 2003; Otsuka et al., 2004) are caused by both the westward-blowing thermospheric winds and, partially, by the Coriolis force effect attributed to the Earth's rotation. Sawako et al. (1980) and Afraimovich et al. (2000a, b) discussed similar phenomena of the LSTIDs of auroral origin. Another explanation of such a structure of a disturbance wave front was proposed by Foster et al. (1989). According to this model, the "curl- 

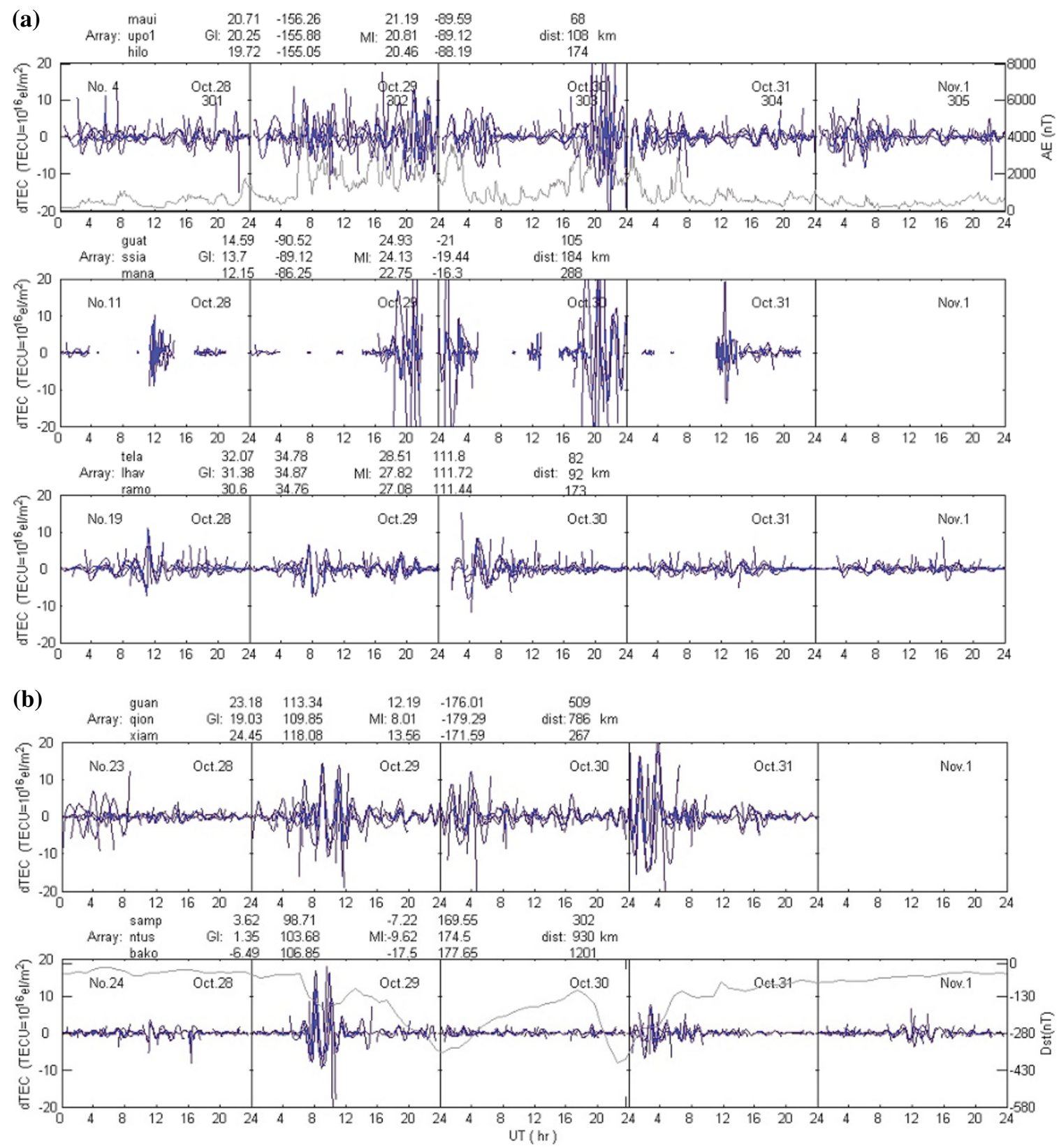

Fig. 7. Variations of dTEC time series at low latitudes $\left(-10^{\circ}\right.$ to $\left.30^{\circ}\right)$ during the multiple magnetic storms; there are no data at arrays 11 and 23 on November 1, 2003.

ing' of the disturbance front is an effect of a great stream of plasma being ejected from the rotating sun-ward polar cap. In addition, Hernández et al. (2006) found that North American and New Zealand MSTIDs have a drift on the propagation azimuth caused by the Coriolis effect. We obtained the same results at both areas. Lin et al. (2005) suggested that the very large fountain effect increased the larger TEC in both hemispheres and that TEC extended to polar regions and propagated westward in the subauroral region due to a poleward electric field in the subauroral polarization stream (SAPS). However, it is still difficult to elaborate on the characteristics of their propagation direction from these observational results due to their large and variable deviation.

As can be seen in the observational results of our study from arrays 4, 11, 19, 23, and 24 at low latitudes shown in Fig. 7, the disturbances happened before the day of the mag- netic storm's SC and lasted from October 28 to November 1 (Doy: 301st to 305th) in varying degrees. The disturbances correlated only slightly with the storm, but they correlated well with the ionospheric inclination that has a large TEC gradient and abundant electrodynamic processes in the low latitudes region, if they were not caused by the TEC error in the measurements in this region.

In addition, from the perturbation time series of dTEC observed at six GPS arrays $(13,16-20)$ shown in Fig. 8, we noticed an abrupt increase disturbance, similar to the SC of the storm, at 9000-1200 UT between $0^{\circ}$ and $75^{\circ}$ of east longitude. Further investigation is needed to clarify the generation mechanism of the sharp phenomena.

\subsection{The Southern Hemisphere}

Due to the limited number of stations and the distribution of these GPS stations in the Southern Hemisphere, it 


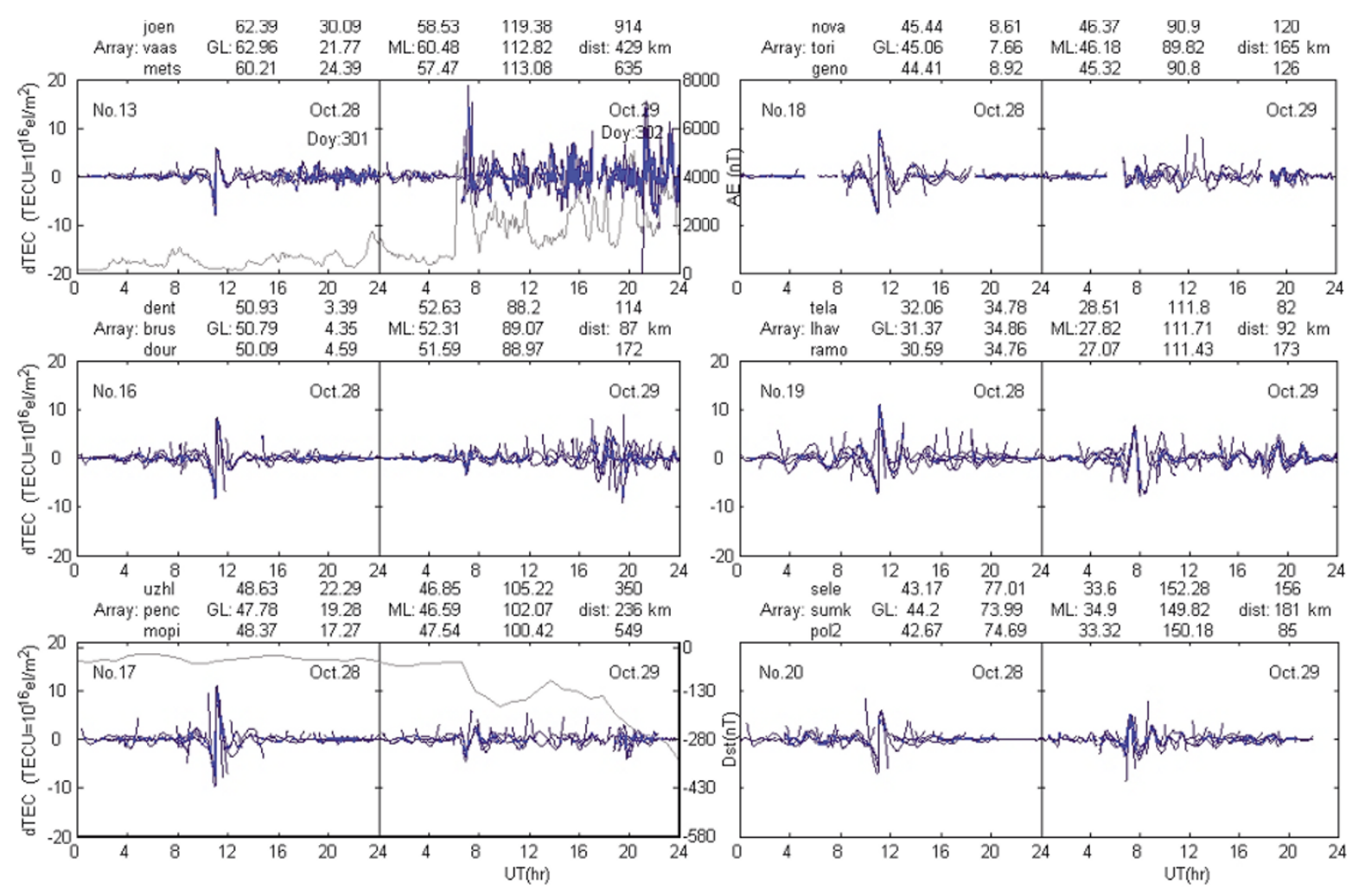

Fig. 8. Variations of dTEC time series between longitudes $0-75^{\circ} \mathrm{E}$ from 301 st to 302 nd . The locations of these arrays are given in Table 1 .

is difficult to determine the precise period and horizontal phase velocity of these LSTIDs in this event. (The maximum distance between stations of one array in the Southern Hemisphere is $5000 \mathrm{~km}$; the minimum distance is $300 \mathrm{~km}$.) We only obtained the range of azimuth, between $320^{\circ}-$ $360^{\circ}-20^{\circ}$, which shows that the ionospheric disturbances are northward propagating from the three selected arrays, 12,25 , and 26 . The northward propagation direction deviated about $30-40^{\circ}$ to the west from array 26 with the influence of the Coriolis force. This result is the same as that in Hernández et al. (2006). We believe that the propagation of ionospheric disturbances in the Southern and Northern Hemisphere is conjugated (Otsuka et al., 2004; Lin et al., 2005; Shiokawa et al., 2005) based on the above facts.

\subsection{Phase velocity of TIDs during the storms}

Several scale irregularities were detected, and an increase in the intensity of medium-scale and small-scale irregularities was caused by the process of dissipation of some intense LSTIDs of auroral origin during the storms (Afraimovich et al., 2006). Ionospheric irregularities (including MSTIDs and small-scale ones) of different scales showed a wide range of horizontal phase velocity (Vph). The follow statements are based on the Vph of TIDs, as is shown in Table 1 and Fig. 4:

1. In the North American region, the dominant Vph deduced from arrays 1, 2, 3, 5, and 6 during the first and second storms shows that both LSTIDs and MSTIDs prevailed at mid-latitude. The dominant $\mathrm{Vph}$ from arrays 8 and 9 was statistically smaller during the second and third storm than that during the first storm. During the third storm, the Vph was dominated by MSTIDs with magnitudes of about $200 \pm 100 \mathrm{~m} / \mathrm{s}$.
2. In the European region at middle latitudes, the Vph derived from arrays $15,16,17$, and 18 was also characterized by both LSTIDs and MSTIDs during the first and second storm. The dominant Vph was decreased to less than 300 $\mathrm{m} / \mathrm{s}$ during the third storm.

3. In the Asian-Australian region at the middle to low latitudes, at arrays 20,21, 22, and 23, the dominant Vph featured with LSTIDs and showed a tendency towards a reduced magnitude with decreasing latitude during the first storm. At the equatorial area, the $\mathrm{Vph}$ deduced from array 24 was dominated by LSTIDs that possibly originated from the Southern Hemisphere and propagate to the north. During the second and third storms, the dominant Vph was reduced remarkably at mid-latitudes, as illustrated at arrays $20-22$, but it remained fairly constant $(\sim 400 \mathrm{~m} / \mathrm{s})$ at array 23. The distribution of azimuth for this array shows that some part of this Vph was contributed from the disturbances that propagated from the Southern Hemisphere.

\section{Conclusion}

The parameters of wave propagation were derived from the multi-channel maximum entropy spectral analysis of the optimized filters on a GPS TEC time series during the October 2003 storms. Based on the statistical results, we studied the propagation and distribution of LSTIDs and MSTIDs in different global sectors, and reached the following conclusions:

1. After the SC of storms on October 29, the obvious ionospheric disturbances were detected over the entire globe, and the amplitudes of ionosphere disturbances at latitudes $60-75^{\circ} \mathrm{N}$ were significantly greater than those in the region at latitudes $50-60^{\circ} \mathrm{N}$. Spectral analysis indicated that 
the average period, horizontal phase velocity, direction of dominant propagation, and life time of ionosphere disturbances are around $55 \pm 5 \mathrm{~min}, 200 \pm 200 \mathrm{~m} / \mathrm{s}, 180^{\circ}$, and $12-$ $16 \mathrm{~h}$, respectively.

2. In the mid-latitudes, there is a high correlation between the time series of ionospheric disturbances and variations in the Dst and AE index. The amplitudes of the disturbances in the North American sector were greater than those in Europe and Asia. The ionospheric disturbances were observed in North America with an average period of $46 \mathrm{~min}$. The dominant $\mathrm{Vph}$ during the first and second storms shows that both LSTIDs and MSTIDs prevailed at mid-latitude. However, the dominant Vph at the arrays 8 and 9 was statistically smaller during the second and third storm than that during the first storm. During the third storm, the Vph was dominated by MSTIDs with magnitudes of about $200 \pm 100 \mathrm{~m} / \mathrm{s}$. There was an obvious dominating southwestward direction for ionospheric disturbances, with an average azimuth of $187.6^{\circ}$ and a southward propagation direction that deviated about $7^{\circ}$ to the west. In addition, the amplitudes of the disturbances in Europe were larger than those in Asia. TIDs observed in Europe were characterized by both LSTIDs and MSTIDs during the first and second storm with a Vph of 100-800 m/s and the dominant azimuth of $184^{\circ}$. The dominant $\mathrm{Vph}$ decreased to less than $300 \mathrm{~m} / \mathrm{s}$ during the third storm. In Asia, the dominant Vph was $400-600 \mathrm{~m} / \mathrm{s}$ during the first storm, and the dominant Vph ( 200-300 m/s) decreased remarkably during the second and third storms, with an average azimuth of $187.5^{\circ}$, respectively. The deflections to the west in the southward propagating disturbances might be subject to the influence of the Coriolis effect.

3. The statistical result in this study reveals that the amplitudes of ionospheric disturbances can reach approximately 13-45 TECU in the low latitudes and equatorial regions and that the durations are longer than those in the middle and high latitudes. The ionospheric disturbances of the middle and low latitudes in the Eastern Hemisphere occurred before the SC of the storms. The average period is around $45 \mathrm{~min}$. The two dominant propagation directions, $215^{\circ}$ and $35^{\circ}$, can be observed in the low latitudes and equatorial region. The southward propagation direction is deflected by $35^{\circ}$ to the west, while the propagation direction of the northward disturbances is deflected by $35 \pm 20^{\circ}$ to the east. The deflection to west may be partially caused by the Coriolis force. Conjugatedly, the azimuth of ionospheric disturbances in the Southern Hemisphere, which is between $320^{\circ}-360^{\circ}-20^{\circ}$, is calculated. Because of the influence of the Coriolis force, the northern propagating disturbances are deflected $30-40^{\circ}$ to west from array 26 .

Acknowledgments. This work is supported by the National Natural Science Foundation of China (grants 40304011 and 40636032), the KIP Pilot Project (kzcx2-yw-123) of Chinese Academy of Science, and the National Important basic Research Project (2006CB806306).

\section{References}

Afraimovich, E. L., E. A. Kosogorov, L. A. Leonovich, K. S. Palamartchouk, N. P. Perevalova, and O. M. Pirog, Determining parameters of large-scale traveling ionospheric disturbances of auroral origin using
GPS-arrays, J. Atmos. Terr. Phys., 62, 553-565, 2000a.

Afraimovich, E. L., E. A. Kosogorov, L. A. Leonovich, K. S. Palamartchouk, N. P. Perevalova, and O. M. Pirog, Observation of largescale traveling ionospheric disturbances of auroral origin by global GPS networks, Earth Planets Space, 52, 669-674, 2000b.

Afraimovich, E. L., E. A. Kosogorov, O. S. Lesyuta and I. I. Ushakov, Geomagnetic Control of the Spectrum of Traveling Ionospheric Disturbances Based on Data from a Global GPS Network, Radiophysics and Quantum Electronics, 44(10), 763-773, doi: 10.1023/A:1013760814426, 2001.

Afraimovich, E. L., E. I. Astafieva, and S. V. Voyeikov, Generation of ionospheric irregularities upon propagation of solitary internal gravity wave during the major magnetic storm of October 29-31, 2003, Radiophys. Quantum Electronics, 49(2), 79-92, 2006.

Akinori, S., F-region radio and optical measurement of nighttime TID campaign, Earth Planets Space, 54, i-ii, 2002.

Beutler G., M. Rothacher, S. Schaer, T. A. Springer, J. Kouba, and R. E. Neilan, The International GPS Service (IGS): An Interdisciplinary Service in Support of Earth Sciences, Adv. Space Res, 23(4), pp. 631635, 1999.

Davies, K., Recent progress in satellite radio beacon studies with particular emphasis on the AST-6 radio beacon experiment, Space Sci.Rev., 25(4), 357-430, 1980.

Ding, F., H. Yuan, W. X. Wan, I. M. Reid, and J. M. Woithe, Occurrence characteristics of medium-scale gravity waves observed in $\mathrm{OH}$ and OI nightglow over Adelaide $\left(34.5^{\circ} \mathrm{S}, 138.5^{\circ} \mathrm{E}\right)$, J. Geophys. Res., 109(D14), 104, doi:10.1029/2003JD004096, 2004.

Foster, J. C., T. Turunen, P. Pollari, H. Kohl, and V. B. Wickwar, Multiradar mapping of auroral convection, Advances in Space Research, 9(5): 19-27, 1989.

Foster, J. C. and W. Rideout, Midlatitude TEC enhancements during the October 2003 superstorm, Geophys. Res. Lett., 32(L12S04), doi:10.1029/2004GL021719, 2005.

Fukao, S., M. C. Kelley, T. Shirakawa, T. Takami, M. Yamamoto, T. Tsuda, and S. Kato, Turbulent upwelling of the mid-latitude ionosphere 1. Observational results by the MU radar, J. Geophys. Res., 96, 37253746, 1991.

Garcia, F. J., M. C. Kelley, J. J. Makela, and C.-S. Huang, Airglow observations of mesoscale low-velocity traveling ionospheric disturbances at midlatitudes, J. Geophys. Res., 105, 18,407-18,415, 2000.

Hernández-Pajares, M., J. M. Juan, and J. Sanz, Medium-scale traveling ionospheric disturbances affecting GPS measurements: Spatial and temporal analysis, J. Geophys. Res., 111(A07S11), doi:10.1029/ 2005JA011474, 2006

Hines, C. O., Internal atmospheric gravity waves at ionospheric heights, Can.J. Phys., 38, 1441-1481, 1960.

Ho, C. M., A. J. Mannucci, U. J. Lindqwister, X. Pi, and B. T. Tsurutani, Global ionosphere perturbations monitored by the worldwide GPS network, Geophys. Res. Lett., 25(22), 3219-3222, 1996.

Hocke, K. and K. Schlegel, A review of atmospheric gravity waves and traveling ionospheric disturbances: 1982-1995, Ann. Geophys., 14, 917-940, 1996.

Hooke, W. H., Ionospheric irregularities produced by internal atmospheric gravity waves, J. Atmos. Terr. Phys., 30, 795, 1968.

Hunsucker, R. D., Atmospheric gravity waves generated in the highlatitude ionosphere: A review, Rev. Geophys., 20, 293-315, 1982.

Kirchengast, G., K. Hocke, and K. Schlegel, The gravity wave-TID relationship: insight via theoretical model-EISCAT data comparison, $J$. Atmos. Terr. Phys., 58(1-4), 233-243, 1996.

Lin, C. H., A. D. Richmond, J. Y. Liu, H. C. Yeh, L. J. Paxton, G. Lu, H. F. Tsai, and S.-Y. Su, Large-scale variations of the low-latitude ionosphere during the October-November 2003 superstorm: Observational results, J. Geophys. Res., 110(A09S28), doi:10.1029/2004JA010900, 2005.

Ogawa, T., N. Balan, Y. Otsuka, K. Shiokawa, C. Ihara, T. Shimomai, and A. Saito, Observations and modeling of $630 \mathrm{~nm}$ airglow and total electron content associated with traveling ionospheric disturbances over Shigaraki, Japan, Earth Planets Space, 54, 45-56, 2002.

Otsuka, Y., K. Shiokawa, T. Ogawa, and P. Wilkinson, Geomagnetic conjugate observations of medium-scale traveling ionospheric disturbances at midlatitude using all-sky airglow imagers, Geophys. Res. Lett., L15803, doi:10.1029/2004GL020262, 2004.

Saito, A., S. Fukao, and S. Miyazaki, High resolution mapping of TEC perturbations with the GSI GPS network over Japan, Geophys. Res. Lett., 25(16), 3079-3082, 1998.

Sawako, M. and H. Shun, Transmission of large-scale TIDs in the ionospheric F2-region, J. Atmos. Terr. Phys, 42, 853-859, 1980. 
Shiokawa, K., Y. Otsuka, M. K. Ejiri, Y. Sahai, T. Kadota, C. Ihara1, T. Ogawa, K. Igarashi, S. Miyazaki, and A. Saito, Imaging observations of the equatorward limit of midlatitude traveling ionospheric disturbances, Earth Planets Space, 54, 57-62, 2002.

Shiokawa, K., C. Ihara, Y. Otsuka, and T. Ogawa, Statistical study of nighttime medium-scale traveling ionospheric disturbances using midlatitude airglow images, J. Geophys. Res., 108(A1), 1052, 2003.

Shiokawa, K., Y. Otsuka, T. Tsugawa, T. Ogawa, A. Saito, K. Ohshima, M. Kubota, T. Maruyama, T. Nakamura, M. Yamamoto, and P. Wilkinson., Geomagnetic conjugate observation of nighttime medium-scale and large-scale traveling ionospheric disturbances: FRONT3 campaign, J. Geophys. Res., 110(A05303), doi:10.1029/2004JA010845, 2005.

Strand, O. N., Multichannel complex maximum entropy (autoregressive) spectral analysis, IEEE Trans. Autom. Control, 22(4), 634-640, 1977.

Tsugawa, T. and A. Saito, A statistical study of large-scale traveling ionospheric disturbances using the GPS network in Japan, J. Geophys. Res., 109, A06302, doi:10.1029/2003JA010302, 2004.

Tsugawa, T., A. Saito, Y. Otsuka, and M. Yamamoto, Damping of largescale traveling ionospheric disturbances detected with GPS networks during the geomagnetic storm, J. Geophys. Res., 108(A3), 1127, 2003. Wan, W. X., J. Li, Z. M. Zhang, and B. W.Reinisch, Study of ionospheric gravity wave disturbances from drift measurements of a digisonde, Chinese J. Geophys., 36(5), 561-569, 1995 (in Chinese).

Wan, W. X., B. Q. Ning, H. Yuan, J. N. Li, L. Li, and J. Liang, TID observation using a short baseline network of GPS receivers, Acta Geodyn. Geophys. Hung., 32(3-4), 321-327, 1997.

Wan, W. X., H. Yuan, B. Q. Ning, J. Liang, and F. Ding, Traveling ionospheric disturbances associated with the tropospheric vortexes around Qinghai-Tibet Plateau, Geophys. Res. Lett., 25(20), 3775-3778, 1998.

Zhao, B., W. Wan, and L. Liu, Responses of equatorial anomaly to the October-November 2003 superstorms, Ann. Geophys., 23, 693-706, 2005 .

M. Wang (e-mail: wangmin@mail.igcas.ac.cn), F. Ding (e-mail: dingf@mail.igcas.ac.cn), W. Wan (e-mail: wanw@mail.igcas.ac.cn), B. Ning (e-mail: nbq@mail.igcas.ac.cn), and B. Zhao 